\title{
1 In foetal development
}

A There are 6 visceral arches and 5 visceral clefts.

B The facial nerve supplies the second or hyoid arch.

C The pharyngeal pouches are formed from the ectodermal furrows.

D The tuberculum impar, the lingual swellings and the hypobranchial eminence form the tongue.

$E$ In the adult the sulcus terminalis in the tongue marks the site of the thyroid rudiment.

\section{Development of the foetal ear}

A The eustachian tube is formed from the ectoderm of the first visceral cleft.

B The auricle develops from the first visceral cleft as a series of 6 tubercles.

C The stapes footplate is derived from ectoderm.

D The inner ear is developed from ectoderm and has reached full size by the 4 th foetal month.

E The stapes superstructure, styloid process and hyoid are derived from the 1st visceral arch.

\section{Development of the temporal bone}

A The tympanic ring and squama are ossified in cartilage.

B The foramen of Huschke is a defect in the tympanic ring.

C Ossification of the endosteal layer of the petromastoid may be defective particularly in the vicinity of the fissula ante fenestram.

D The facial nerve is well protected at birth by the mastoid process.

E In the infant the mastoid antrum lies below the tympanic cavity and about $5 \mathrm{~mm}$ deep to the bony surface. 